\title{
Estudio cuasi-experimental en la aplicación de metodos cooperativos para la enseñanza de la asignatura en filosofia
}

\section{Resumen}

La investigación se realizó con 62 adolescentes (mujeres y hombres) de educación media vocacional del grado décimo, de los cuales 29 corresponden al grado décimo B y 33 al grado décimo A del colegio de Nuestra Señora de las Mercedes del Municipio de Sardinata, por medio de un diseño cuasi- experimental con un grupo experimental, un grupo control y con pruebas post-tes, en los dos grupos se investigó la influencia que en el aprendizaje de concepto y rendimiento académico tiene la aplicación de métodos cooperativos para la enseñanza de la asignatura en filosofía. El grado de conceptualización y rendimiento académico fue medido mediante pruebas - postest con una confiabilidad de $80 \%$ la cual se obtuvo mediante método de mitades partidas. Se aplico la prueba de Shapiro-Wilk para muestras menores de 50 y se encontró, previa validación de los supuestos de normalidad, A demás las distribuciones de las calificaciones de los dos grupos presentan la misma varianza con un valor de $p=0.073$ en la prueba de Levene y que existe una diferencia significativa entre las calificaciones de los dos grupos ( $p=$ 0.000 prueba t).

Palabras clave: estrategias metodológicas, método cooperativo, normalidad

\section{Abstract}

We did the investigation with 62 students (men and women), they study at Nuestra Señora de las Mercedes School in Sardinata, they are in 10th grade; 29 of these students are in $10 \mathrm{~B}$ grade and the other 33 are in $10 \mathrm{~A}$ grade. By an experimental designed with an experimental group, a group of control and using some qualified post-test in both grades; we realized how important is learning a topic and improving the academic performance by using cooperative methods to teach this subject in Philosophy We measured the rank of getting the topic and improving the academic performance by applying some post-tests with $80 \%$ of reliability, we got this result by using the divided Halves method, we applied the Shapiro-Wilk test for samples fewer than 50\% and we found Previous values of the normal assumptions, and the grades of these two groups show the same variance with a value of $p=0.073$ on the Levene test and it has a very important diference between the different grades of the two groups $(p=0.000$ prueba $t)$.

Key Words: methodoligic strategies, cooperative method and the normal way

\footnotetext{
${ }^{1}$ Ingeniero Civil. Especialista en estadística Aplicada. Universidad Francisco de Paula Santander. Departamento de Matemáticas y estadística. Carmenmedrano@ufps.edu.co

${ }^{2}$ Licenciado en Filosofía. Colegio de Nuestra Señora de las Mercedes municipio de Sardinata. araque8302@hotmail.com
} 


\section{Introducción}

U na preocupación básica para el docente es descubrir cuáles son los elementos que hacen que su clase funcione o no, que el ambiente de aprendizaje que ha creado sea eficaz en relación a los objetivos formativos que se ha propuesto [1], por eso abordar el tema sobre aplicación de nuevas metodología de enseñanza, implica atreverse a romper miradas educativas, a destruir prejuicios, a realizar giros en cuanto a nuevas comprensiones en la actividad que cumple la educación, así mismo lograr percibir que metodología es la más acertada y que de mejores resultados ayuda a identificar nuevas variables de trabajo pedagógico y sicológico.

El presente trabajo investigó un aspecto particular en el proceso de enseñanza - aprendizaje de la asignatura en filosofía en los grados décimo de la Institución educativa Nuestra Señora de las Mercedes del Municipio de Sardinata, se utilizó una metodología de enseñanza basada en métodos de auto-aprendizaje cooperativo en el grupo experimental, para luego comparar los resultados obtenidos en las evaluaciones con un grupo control que desarrolló la asignatura a través de la clase magistral como metodología de enseñanza, con el fin de mejorar el grado de conceptualización y aprendizaje de los temas de: La escolástica, Santo Tomas de Aquino, San Agustín de Hipona.

Este trabajo intento, sin mayores pretensiones, establecer si el uso de nueva metodología de enseñanza- aprendizaje puede influir significativamente en el aprendizaje de algunos conceptos filosóficos en los estudiantes de décimo grado.

\section{Aprendizaje Cooperativo}

Bajo el concepto de aprendizaje cooperativo se engloba un conjunto muy diferente de técnicas y métodos que a veces tienen poco en común y suelen diferenciarse en cuanto al grado de interdependencia de las recompensas, grado de interdependencia de la tarea, grado de responsabilidad individual, grado de estructura impuesta por el profesor o por la propia tarea y grado de utilización de la competición que puede llegar hasta la no competición. (Fabra, 1992) [2].

\section{Materiales y Métodos}

Tipo de investigación: Es una investigación de tipo cuasi-experimental pues los sujetos no son asignados al azar a los grupos ni emparejados, sino que dichos grupos ya estaban formados antes del experimento [3].

Diseño: El proyecto utilizó un diseño con dos grupos (experimental y control) y dos mediciones posterior al tratamiento. El curso décimo $\mathrm{A}$, desarrollo los temas seleccionados de la asignatura en filosofía por medio de una metodología de auto-aprendizaje cooperativo, este grupo fue llamado grupo experimental y el curso decimo B que desarrollo la asignatura en filosofía con clase magistral y se le llamó grupo control. Los grupos son comparados en la post prueba para analizar si el tratamiento experimental tuvo un efecto sobre la variable dependiente.

\section{Población}

La investigación se realizó en el colegio Nuestra Señora de las Mercedes del Municipio de Sardinata, la mayoría de los estudiantes son jóvenes, algunos desplazados, otros de escasos recursos económicos y otros provienen de la zona rural.

\section{Tamaño de la Muestra}

La investigación se realizó con 62 adolescentes (mujeresyhombres) deeducación mediavocacional del grado décimo, de los cuales 29 corresponden al grado décimo B y 33 al grado decimo A de la Institución Educativa Nuestra Señora de las Mercedes del municipio de Sardinata.

\section{Planteamiento de Hipótesis}

La metodología de enseñanza aplicada en el grupo experimental (décimo A) dan mejores resultados que la metodología tradicional aplicada en el grupo control (decimo B).

\section{Diseño de Instrumentos}

El proceso de construcción de los instrumentos consistió en dos pruebas para cada grupo, en el grupo control los instrumentos siguieron los parámetros de las pruebas realizadas anteriormente, las cuales están compuestas por preguntas abiertas y cuadros comparativos.

Para el grupo experimental se diseñaron pruebas 
con preguntas de respuesta única, donde la preguntas se expresaron en párrafos que suscita al estudiante que analice, interprete y con base en lo leído escoja la repuesta, los temas evaluados en las pruebas fueron: La escolástica, Santo Tomas de Aquino, San Agustín de Hipona.

Las notas de los respectivos instrumentos evaluativos se analizaron según la escala valorativa adoptada por la Institución educativa colegio Nuestra Señora de las Mercedes, que es de la siguiente manera: 0 a 29 Deficiente, 30 a 69 Insuficiente, 70 a 80 Aceptable, 81 a 94 sobresaliente, 95 a 100 Excelente.

\section{Procedimiento.}

En el tercer periodo escolar del año 2009, se inicio la investigación, los temas seleccionados se enseñaron por medio de la metodología de auto-aprendizaje cooperativo a través la de las siguientes etapas: formación sobre el proceso de aprendizaje y objetivos perseguidos, creación de los equipos dentro del grupo, búsqueda e información del texto más adecuado, redacción de preguntas de análisis, elaboración de mapas conceptuales, lectura y comprensión del texto, análisis intergrupal, presentación de las ideas de los equipos, debate y conclusiones.

Se aplicaron las pruebas tanto para el grupo control como para el grupo experimental, luego se realizó la tabulación y análisis de los resultados de los post-test aplicados a los alumnos de los grados décimo A y B, se procedió a realizar los cálculos para determinar la puntuación promedio de los estudiantes en cada prueba y así obtener la puntuación promedio del grupo. Luego de se procedió a realizar una prueba $t$ para dos poblaciones para verificar que los resultados obtenidos no son resultados al azar [4].

Se procedió a establecer la hipótesis estadística nula:

Ho: los métodos de enseñanza producen un aprendizaje idéntico Ho: $\mu \mathrm{A}=\mu \mathrm{B}$, donde $\mu \mathrm{A}$ es la media poblacional de los promedios de las puntuaciones utilizada en el grupo experimental

Ha: La metodología aplicada en el grupo experimental mejora el aprendizaje de la asignatura en filosofía que la metodología aplicada en el grupo control. Ha: $\mu \mathrm{a}>\mu \mathrm{B}$. se trabajo con un nivel de significancia del $5 \%$.

\section{Resultados y discusión}

Figura No 1. Promedio de notas en cada una de las pruebas aplicada a cada uno de los grupos

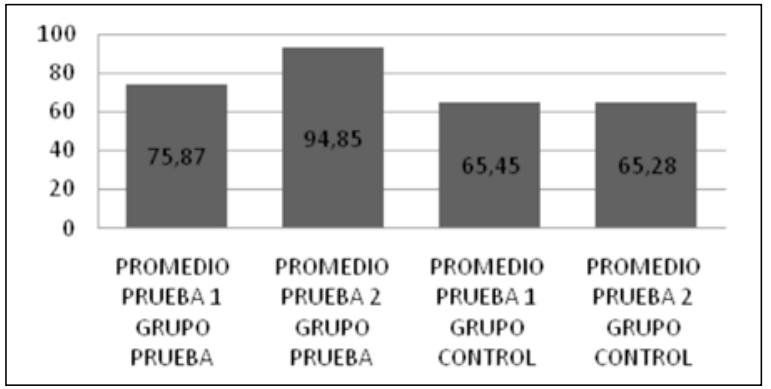

Figura No 2. Desviaciones estándar de las notas en cada uno de los grupos

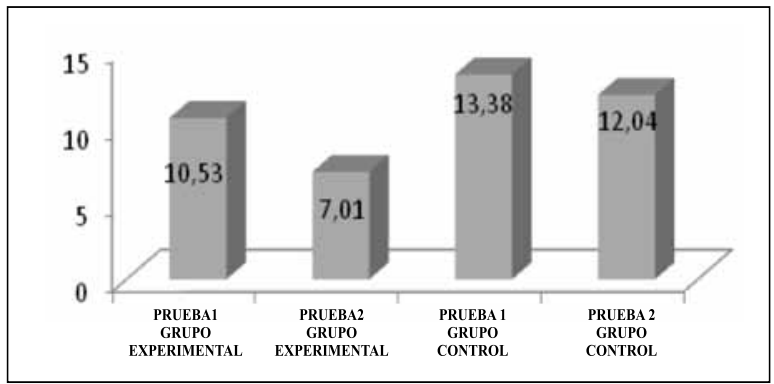

En el grupo experimental los resultados obtenidos en las pruebas muestran que con la nueva metodología las notas promedios de los estudiantes aumentan con relación a los resultados obtenidos en el grupo control, también se puede concluir que en la segunda prueba el grupo experimental logró un aumento en la nota promedio del grupo de manera significativa, mientras que el grupo control no mostró ningún aumento en los resultados.

Igualmente sucedió con la desviación estándar de la nota promedio, está tiende a disminuir en la segunda prueba aplicada al grupo experimental, mientras que el grupo control la desviación estándar se mantiene.

Tabla No 1. Resultados estadísticos de las puntuaciones promedios de los grupos

\begin{tabular}{|c|c|c|c|r|c|}
\hline & $\mathrm{N}$ & Mínimo & Máximo & Media & Desv. típ \\
\hline $\begin{array}{c}\text { Puntuaciones promedios } \\
\text { grupo experimental }\end{array}$ & 33 & 70,00 & 97,00 & 85,3636 & 6,6289 \\
\hline $\begin{array}{c}\text { Puntuaciones promedios } \\
\text { grupo control }\end{array}$ & 29 & 46,50 & 80,00 & 65,4655 & 9,2870 \\
\hline
\end{tabular}


La media de la puntuación promedio en el grupo experimental fue de 85,36 y para el grupo control fue de 65,46. A primera vista estos resultados muestran que el grupo experimental dio mejores resultados que el grupo control, pero también existe la posibilidad que esto resultados pudieron ser obtenidos por mero funcionamiento al azar, por lo tanto se realizó una prueba hipótesis para verificar estos resultados,

Para validar los supuestos de normalidad de las distribuciones, implícitos al aplicar la prueba $t$, se aplicó la prueba no-paramétrica de Shapiro Wilk a las dos distribuciones analizadas pos-test para los dos grupos, experimental y control. Los resultados de la prueba de normalidad indican que las puntuaciones promedios del grupo experimental y del grupo control siguen una distribución normal.

Tabla No 2. Pruebas de normalidad grupo control

\begin{tabular}{|c|c|c|c|}
\hline & \multicolumn{3}{|c|}{ Shapiro-Wilk } \\
\hline & Estadístico & gl & Sig. \\
\hline $\begin{array}{c}\text { puntuación } \\
\text { promedio }\end{array}$ &, 962 & 29 &, 449 \\
\hline
\end{tabular}

Tabla No 3. Prueba de normalidad para el grupo experimental

\begin{tabular}{|c|c|c|c|}
\hline & \multicolumn{3}{|c|}{ Shapiro-Wilk } \\
\hline & Estadístico & gl & Sig. \\
\hline $\begin{array}{c}\text { puntuación } \\
\text { promedio }\end{array}$ &, 950 & 33 &, 216 \\
\hline
\end{tabular}

La prueba Levene para la igualdad de varianzas indica que con una significancia de 0,073 se puede concluir que la varianza de los dos grupos

Tabla No 4. Prueba de hipótesis para diferencias de medias de las puntuaciones

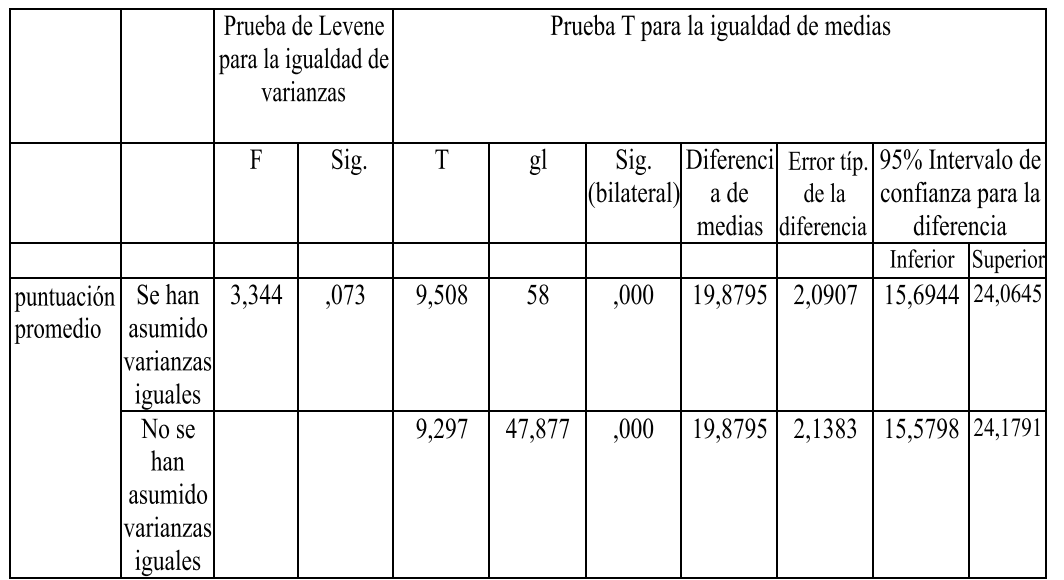

son iguales, con relación a la prueba $\mathrm{T}$ para la igualdad de medias indica que con un valor de significancia de 0,000 se rechaza la hipótesis nula, que significa que la metodología empleada en el grupo experimental mejora el aprendizaje de los estudiantes de la asignatura en filosofía.

\section{Conclusiones}

La investigación por medio del análisis estadístico de los resultados de los post-test aplicados al grupo control y al grupo experimental permitió concluir lo siguiente:

La prueba de Shapiro-Wilk aplicadas a las distribuciones del post-test en los dos grupos, validó el supuesto de normalidad de las distribuciones. Este es un supuesto en la prueba para la significación de la diferencia de medias debido al tratamiento y respecto de los resultados del post- test.

La prueba T de Student aplicada a los resultados del post- test en los dos grupos confirmó la hipótesis de que existe diferencia académica significativa ante el grupo control y el grupo experimental debido al tratamiento experimental aplicado y no debido al azar o a otras variables, es decir que con un nivel de confianza del $95 \%$ podemos afirmar que usando el método de aprendizaje cooperativo se produce en los estudiantes un mejor rendimiento académico y una mejor asimilación de conceptos.

La prueba de Levene aplicada a los resultados del post-test en el grupo experimental y de control valida la hipótesis nula de igualdad de varianzas. La igualdad de varianzas es un supuesto requerido para la prueba de hipótesis de la investigación inmediatamente anterior.

A partir de de los resultados de la investigación se puede deducir que cuando se alcanza a romper esquemas mentales y se adoptan nuevas propuestas pedagógicas y metodológicas se pueden alcanzar buenos resultados; puesto que en ellas se incita no tanto a manejar conceptos de memoria, a pie de 
letra, memorizar una gran extensión de texto. Con las nuevas metodologías lo que se pretende es que el estudiante lea, analice, interprete y con base en lo leído de repuestas, no es memorizar sino análisis e interpretación.

\section{Agradecimientos}

Directivas y estudiantes del colegio Nuestra Señora de las Mercedes del Municipio de Sardinata.,

\section{Bibliografía}

[1] IGLESIAS, Lina María. Observación y evaluación del ambiente de aprendizaje, en revista Iberoamericana de Educación. No 47. Mayo de 2008

[2] FABRA, M.L. El trabajo cooperativo revisión y perspectivas. Aula de Innovación Educativa. 1992.

[3] LEÓN, O.G. y Montero, I. Diseño de Investigaciones. Madrid: Mc Graw Hill. 1997

[4] WALPOLE, Ronald E. y Raymond Myer. Probabilidad y Estadística. Mc Graw Hill. 1999. 